\title{
EXACT FUZZY OBSERVER FOR A BAKER'S YEAST FERMENTATION PROCESS
}

\author{
Enrique Herrera $^{a, 1}$, Bernardino Castillo ${ }^{a}$, Jesús Ramírez ${ }^{b}$ and Eugénio C. Ferreira ${ }^{c}$ \\ ${ }^{a}$ Centro de Investigación y de Estudios Avanzados del I.P.N. Unidad Guadalajara, \\ Av. Cientifica 1145, Colonia el bajío C.P. 45010, Zapopan, México \\ ${ }^{b}$ Centro de Investigación y Asistencia en Tecnología y Diseño del Estado de Jalisco A.C. \\ Av Normalistas 800, C.P. 44270, Guadalajara, México \\ ${ }^{c} I B B$ Institute for Biotechnology and Bioengineering, Centre of Biological Engineering, \\ Universidade do Minho 4710-057,Braga Portugal
}

toledo@gdl.cinvestav.mx,\{eherrera,jramirez\}@ciatej.net.mx,ecferreira@deb.uminho.pt

\begin{abstract}
The purpose of this work is to design an exact fuzzy observer for a bioprocess switching between two different metabolic states. A continuous baker's yeast culture is divided in two sub-models: a respiro-fermentative with ethanol production and a respirative with ethanol consumption. An exact fuzzy observer model using sector nonlinearity was built for both nonlinear models; the observer gains were designed using Linear Matrix Inequalities (LMI's). The observer premise variables depend on the state variables estimated by the fuzzy observer. Copyright (C) 2007 IFAC
\end{abstract}

Keywords: Nonlinear system, Fuzzy models, State estimation, Fermentation processes.

\section{INTRODUCTION}

In bioindustries producing food or beverages by using bioprocesses, there are strong demands of systems providing optimization and automatic control of those bioprocesses in terms of quality control and cost savings (Horiouchi, et al., 2002). However, the lack of cheap and reliable sensors providing on line measurements of the biological state variables has hampered the application of automatic control to bioprocess (Bastin and Dochain, 1990). This situation encourages the searching of new software sensors in bioprocesses.

Two class of state observers for (bio)chemical processes can be found in the literature (Dochain, 2003). A first class of observer called asymptotic observer, is based on the idea that the uncertainty in

${ }^{1} \mathrm{On} \mathrm{Ph}$. D. studies leave from the Centro de Investigación y Asistencia en Tecnología y Diseño del Estado de Jalisco A. C. bioprocess models is located in the process kinetics models. A second class is based on the perfect knowledge of the model structure (Luenberger, Kalman Observers and nonlinear observers). When the perfect knowledge of a nonlinear system is available, also a fuzzy model can be used. A first approach can be done using the Takagi-Sugeno (TS) fuzzy model (Takagi and Sugeno, 1985), where the consequent part of the fuzzy rules are replaced by linear systems. This can be attained, for example, linearizing the model around operational points, getting local linear representation of the nonlinear system.

Another way for obtaining TS models can be achieved using the method of sector nonlinearities, which allows constructing an exact fuzzy model from the original nonlinear system by means of linear subsystems (Tanaka and Wang, 2001). From this exact model, a state observer may be designed based on the linear subsystems. Different fuzzy logic applications to bioprocesses can be found in the 
scientific literature (Horiouchi, et al., 1998; Azevedo et al., 2004) among others.

In this work a fuzzy state observer based on sector nonlinearities is proposed and applied to a continuous baker's yeast process. An interesting feature of this model is the splitting in two different partial models: a respire-fermentative (RF) model with ethanol production and the respirative (R) model with ethanol consumption. The switching condition depends on whether the process is consuming or producing ethanol. The observer premise variables depend on the estimated variables by the fuzzy observer. The use of fuzzy observers obtained from an exact fuzzy model, applied to a bioprocess described by partial models has not been, to the best author's knowledge, reported in the literature.

\section{PRELIMINARIES ON FUZZY MODELS}

\subsection{Takagi-Sugeno Fuzzy Model.}

The Takagi-Sugeno fuzzy models are used to represent nonlinear dynamics by means of a set of IF-THEN rules. The consequent part of the rules are local linear systems obtained from specific information about the original nonlinear plant. The $i$ th rule of a continuous fuzzy model has the following form:

Rule i:

$$
\begin{aligned}
& \text { IF } z_{1}(t) \text { is } M_{1}^{i} \text { and...and } z_{p}(t) \text { is } M_{p}^{i} \\
& \text { THEN }\left\{\begin{array}{l}
\dot{x}(t)=A_{i} x(t)+B_{i} u(t) \\
y(t)=C_{i} x(t)
\end{array} \quad i=1, \ldots, r .\right.
\end{aligned}
$$

where $M_{j}^{i}$ is a fuzzy set and $r$ is the number of rules in the fuzzy model; $x(t) \in R^{n}$ is the state vector, $u(t) \in R^{m}$ is the input vector, $y(t) \in R^{q}$ is the output vector, $A_{i} \in R^{n x n}, B_{i} \in R^{n x m}$, and $C_{i} \in R^{q x n}$ are suitable matrices, and $z(t)=\left[z_{l}(t), \ldots, z_{p}(t)\right]$ is a known vector of premise variables which may depend partially on the state $x(t)$. Given a pair of $(x(t), u(t))$ and using a product inference engine, the aggregate TS fuzzy model can be inferred as:

$$
\begin{gathered}
\dot{x}(t)=\frac{\sum_{i=1}^{r} \varpi_{i}(z(t))\left\{A_{i} x(t)+B_{i} u(t)\right\}}{\sum_{i=1}^{r} \varpi_{i}(z(t))} \\
=\sum_{i=1}^{r} h_{i}(z(t))\left\{A_{i} x(t)+B_{i} u(t)\right\}, \\
y(t)=\frac{\sum_{i=1}^{r} \varpi(z(t)) C_{i} x(t)}{\sum_{i=1}^{r} \varpi(z(t))} \\
=\sum_{i=1}^{r} h_{i}(z(t)) C_{i} x(t),
\end{gathered}
$$

where

$$
\begin{aligned}
& \varpi_{i}(z(t))=\prod_{j=1}^{p} M_{j}^{i}\left(z_{j}(t)\right), \\
& h_{i}(z(t))=\frac{\varpi_{i}(z(t))}{\sum_{i=1}^{r} \varpi_{i}(z(t))},
\end{aligned}
$$

for all $t$. The term $M_{j}^{i}\left(z_{j}(t)\right)$ is the membership value of $z_{j}(t)$ in $M_{j}^{i}$. Since

$$
\varpi_{i}(z(t)) \geq 0 \text { and } \sum_{i=1}^{r} \varpi_{i}(z(t))>0, \quad i=1, \ldots, r,
$$

we have that

$$
h_{i}(z(t)) \geq 0 \text { and } \sum_{i=1}^{r} h_{i}(z(t))=1, \quad i=1, \ldots r,
$$

for all $t$.

\subsection{Fuzzy Observers}

The state of a system is not always fully available, so it is necessary to use an observer to reconstruct, at least partially the states variables of the process. This requires to satisfy that $\lim _{t \rightarrow 0}(x(t)-\hat{x}(t))=0$, where $\hat{x}(t)$ denotes the state vector estimated by the fuzzy observer. There are two cases for fuzzy observers design depending on whether or not $z(t)$ depends on the state variables estimated by a fuzzy observer (Tanaka and Wang, 2001). Given the TS fuzzy model (1), the $i$ th rule of a continuous fuzzy observer can be constructed as:

$$
\begin{aligned}
& \text { Observer Rule } i \\
& \qquad \begin{array}{l}
\text { IF } z_{1}(t) \text { is } M_{1}^{i} \text { and...and } z_{p}(t) \text { is } M_{p}^{i} \\
\text { THEN } \\
\hat{x}=\sum_{i=1}^{r} h_{i}(z(t))\left\{A_{i} \hat{x}(t)+B_{i} u(t)+K_{i}(y(t)-\hat{y}(t))\right\}, \\
\quad \hat{y}(t)=h_{i}(z(t)) C_{i} \hat{x}(t) .
\end{array}
\end{aligned}
$$

where $K_{i}$ is the observer gain for the $i$ th subsystem. If $z(t)$ depends on the estimated state variables, the observer takes the following form:

$$
\begin{gathered}
\hat{x}=\sum_{i=1}^{r} h_{i}(z(t))\left\{A_{i} \hat{x}(t)+B_{i} u(t)+K_{i}(y(t)-\hat{y}(t))\right\}, \\
\hat{y}(t)=h_{i}(\hat{z}(t)) C_{i} \hat{x}(t) .
\end{gathered}
$$

\section{THE BAKER'S YEAST MODEL}

A continuous baker's yeast culture can be represented by the following set of differential equations

$$
\begin{aligned}
& \dot{x}_{1}=\left(\mu_{s}^{o}+\mu_{s}^{r}+\mu_{e}^{o}\right) x_{1}-D x_{1} \\
& \dot{x}_{2}=\left(-k_{1} \mu_{s}^{o}-k_{2} \mu_{s}^{r}\right) x_{1}-D x_{2}+D S_{i n} \\
& \dot{x}_{3}=\left(k_{3} \mu_{s}^{r}-k_{4} \mu_{e}^{o}\right) x_{1}-D x_{3} \\
& \dot{x}_{4}=\left(-k_{5} \mu_{s}^{o}-k_{6} \mu_{e}^{o}\right) x_{1}-D x_{4}+O T R
\end{aligned}
$$


Pormeleau (1990) suggested a reformulation of model (11) using two partial models: a respirofermentative partial model (RF) with ethanol production and a respirative partial model $(\mathrm{R})$ with ethanol consumption. With this reformulation a split process model is obtained switching from the RF partial model to the $\mathrm{R}$ partial model and vice versa depending on whether the system is consuming or producing ethanol. To precise the ideas consider a nonlinear system described by the model (11) which can be written as:

$$
\begin{gathered}
\dot{x}(t)=f_{i}(x(t))+B u(t)+d(x(t)) \\
y(t)=h(x(t))
\end{gathered}
$$

where $f_{i}(x(t))$ describe both the $\mathrm{RF}$ and $\mathrm{R}$ partial models, namely

$$
f_{1}=\left[\begin{array}{c}
\left(\mu_{s_{-} R F}^{o}+\mu_{s_{-} R F}^{r}\right) x_{1}-D x_{1} \\
\left(-k_{1} \mu_{s_{-} R F}^{o}-k_{2} \mu_{s_{-} R F}^{r}\right) x_{1}-D x_{2} \\
k_{3} \mu_{s_{-} R F}^{r} x_{1}-D x_{3} \\
-k_{5} \mu_{s_{-} R F}^{o} x_{1}-D x_{4}
\end{array}\right]:=f_{R F}
$$

and for the R model

$$
f_{2}=\left[\begin{array}{c}
\left(\mu_{s_{-} R}^{o}+\mu_{e_{-} R}^{o}\right) x_{1}-D x_{1} \\
-k_{1} \mu_{s_{-} R}^{o} x_{1}-D x_{2} \\
-k_{4} \mu_{e_{-} R}^{o} x_{1}-D x_{3} \\
\left(-k_{5} \mu_{s_{-} R}^{o}-k_{6} \mu_{e_{-} R}^{o}\right) x_{1}-D x_{4}
\end{array}\right]:=f_{R}
$$

and $u(t)=S_{i n}, \quad B=\left[\begin{array}{llll}0 & D & 0 & 0\end{array}\right]^{T}$ and $h(x(t))=x_{3}$. The oxygen transfer rate OTR is assumed to be a measurable and known perturbation, and thus

$$
d=\left[\begin{array}{llll}
0 & 0 & 0 & O T R
\end{array}\right]^{T}
$$

The variables and parameter values used in (11-14) are shown in table 1. Yield coefficients values for $k_{1}$,

\begin{tabular}{|c|c|c|}
\hline Variab & ole / parameter & Inits \\
\hline$x_{1}$ & $=$ biomass & $\mathrm{g} / 1$ \\
\hline$x_{2}$ & $=$ substrate & $\mathrm{g} / 1$ \\
\hline & $=$ ethanol & $\mathrm{g} / 1$ \\
\hline$x_{4}$ & $=$ dissolved oxygen & $\mathrm{mg} / 1$ \\
\hline$S_{i n}$ & $=$ inlet substrate concentration & $\mathrm{g} / 1$ \\
\hline$D=$ & $=$ dilution rate & $h^{-1}$ \\
\hline & $\begin{aligned}= & \text { specific growth rate for respi } \\
& \text { growth on glucose }\end{aligned}$ & $h^{-1}$ \\
\hline & $\begin{aligned}= & \text { specific growth rate for ferm } \\
& \text { growth on glucose }\end{aligned}$ & $h^{-1}$ \\
\hline & $\begin{aligned}= & \text { specific growth rate for respir } \\
& \text { growth on ethanol. }\end{aligned}$ & $\mathrm{h}^{-1}$ \\
\hline
\end{tabular}
$k_{2}, k_{3}, k_{4}, k_{5}, k_{6}$ and the oxygen transfer rate (OTR) are described in Ferreira, (1995)

Table 1 Parameters and variables used in the baker's yeast model.
The specific rates for the RF partial model are given by:

$$
\begin{gathered}
\mu_{s_{-} R F}^{o}=Y_{O 2}\left(q_{o}^{\max } \frac{x_{4}}{K o+x_{4}}\right) \\
\mu_{s_{-} R F}^{r}=y_{r}\left(q_{s}^{\max } \frac{x_{2}}{K s+x_{2}}-q_{o}^{\max } \frac{x_{4}}{K o+x_{4}} \frac{Y_{O 2}}{Y_{O}}\right) .
\end{gathered}
$$

For the $\mathrm{R}$ partial model the specific rates are given by

$$
\mu_{s_{-} R}^{o}=Y_{O}\left(q_{s}^{\max } \frac{x_{2}}{K s+x_{2}}\right),
$$

and $\mu_{e_{-} R}^{o}$, which can take the following values

$$
\mu_{\mathrm{e}_{-} \mathrm{R}}^{\mathrm{o}}=\left\{\begin{array}{lll}
q_{e_{1}} & I F & q_{e_{1}}<q_{e_{2}} \\
q_{e_{2}} & I F & q_{e_{1}} \geq q_{e_{2}}
\end{array}\right.
$$

where

$$
\begin{gathered}
q_{e_{1}}=Y_{e} q_{e}^{\max } \frac{x_{3}}{K e+x_{3}} \frac{K i}{K i+x_{2}} \\
q_{e_{2}}=Y_{O 2} e\left(q_{o}^{\max } \frac{x_{4}}{K o+x_{4}}-\frac{Y_{O}}{Y_{O 2}}\left(q_{s}^{\max } \frac{x_{2}}{K s+x_{2}}\right)\right)
\end{gathered}
$$

From equation (20) and (21) it can be inferred that the R partial model given by (14) should be split in two new models: (Rqe1) when $\mu_{e R}^{o}=q_{e_{1}}$ and (Rqe2) when $\mu_{e_{-} R}^{o}=q_{e_{2}}$. The switching condition between the RF and R partial model is described by Ferreira, (1995). The yield coefficients used in the specific rates (16-21) and in the partial models (13) and (14) are those reported by Sonnleitner and Käppeli (1986). To change between the RF to the $\mathrm{R}$ models, $S_{i n}$ was varied according to figure 1 , while the dilution rate was set to $0.2 \mathrm{~h}^{-1}$.

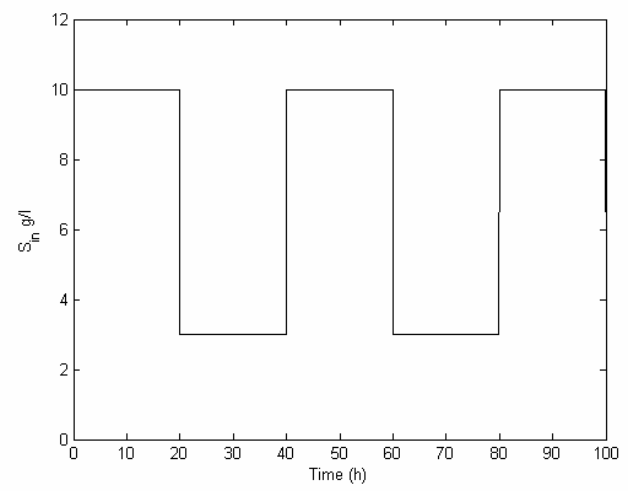

Fig. 1. The inlet substrate concentration signal

\section{THE EXACT FUZZY MODEL}

When the nonlinear dynamic model for the baker's yeast is known, as well as all their parameters, a fuzzy exact model can be derived from the given nonlinear model. This requires a sector nonlinearity 
approach (Tanaka and Wang, 2001). To construct the $\mathrm{RF}$ and $\mathrm{R}$ exact fuzzy models, $f_{R F}$ can be expressed as

RF model

$f_{R F}=\left[\begin{array}{cccc}\frac{x_{4}}{K o+x_{4}} q_{o}^{\max }\left(Y_{O 2}-Y_{r} \frac{Y_{O 2}}{Y_{O}}\right)-D & Y_{r} q_{s}^{\max } \frac{x_{1}}{K s+x_{2}} & 0 & 0 \\ \frac{x_{4}}{K o+x_{4}} q_{o}^{\max }\left(-k_{1} Y_{O 2}+k_{2} Y_{r} \frac{Y_{O 2}}{Y_{O}}\right) & -k_{2} Y_{r} q_{s}^{\max } \frac{x_{1}}{K s+x_{2}}-D & 0 & 0 \\ -k_{3} Y_{r} q_{o x}^{\max } \frac{Y_{O 2}}{Y_{O}} \frac{x_{4}}{K o+x_{4}} & k_{3} Y_{r} q_{s}^{\max } \frac{x_{1}}{K s+x_{2}} & -D & 0 \\ -k_{5} Y_{O_{2}} q_{o}^{\max } \frac{x_{4}}{K o+x_{4}} & 0 & 0 & -D\end{array}\right]\left[\begin{array}{l}x_{1} \\ x_{2} \\ x_{3} \\ x_{4}\end{array}\right]$,

and according to (20) and (21) $f_{R}$ should be split to

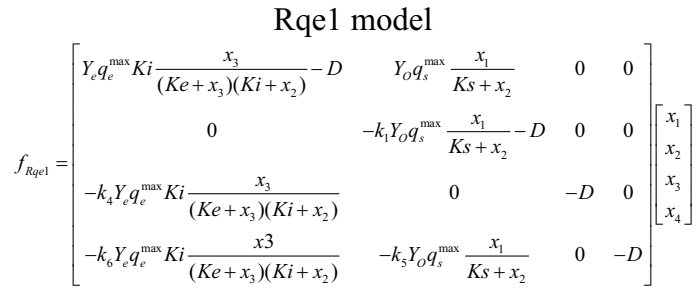

$$
f_{R, q 2}=\left[\begin{array}{cccc}
Y_{O 2} e q_{o}^{\max } \frac{x_{4}}{K o+x_{4}}-D & \frac{x_{1}}{K s+x_{2}} q_{s}^{\max }\left(Y_{O}-Y_{O 2} e \frac{Y_{O}}{Y_{O 2}}\right) & 0 & 0 \\
0 & -k_{1} Y_{O} q_{s}^{\max } \frac{x_{1}}{K s+x_{2}}-D & 0 & 0 \\
-k_{4} Y_{O 2} e q_{o}^{\max } \frac{x_{4}}{K o+x_{4}} & k_{4} Y_{O 2} e q_{s}^{\max } \frac{x_{1}}{K s+x_{2}} \frac{Y_{O}}{Y_{O 2}} & -D & 0 \\
-k_{6} Y_{O 2} e q_{o}^{\max } \frac{x_{4}}{K o+x_{4}} & \frac{x_{1}}{K s+x_{2}} q_{s}^{\max }\left(-k_{5} Y_{O}+k_{6} Y_{O 2} e \frac{Y_{O}}{Y_{O 2}}\right) & 0 & -D
\end{array}\right]\left[\begin{array}{c}
x_{1} \\
x_{2} \\
x_{3} \\
x_{4}
\end{array}\right]
$$

From (22-24) the fuzzy exact model using sector nonlinearities can be constructed. The premise variables are chosen as:

$$
\begin{gathered}
z_{1}(t)=\frac{x_{4}}{K o+x_{4}}(25) \quad z_{2}(t)=\frac{x_{1}}{K s+x_{2}} \\
z_{3}(t)=\frac{x_{3}}{\left(K e+x_{2}\right)\left(K i+x_{3}\right)} .
\end{gathered}
$$

To construct the membership functions we use

$$
z_{1}(t)=\sum_{i=1}^{2} M_{i}\left(z_{1}(t)\right) a_{i}
$$

where the property $M_{1}\left(z_{1}(t)\right)+M_{2}\left(z_{1}(t)\right)=1$ must be accomplished (Tanaka and Wang, 2001). The membership functions for the RF partial model can be built from the premise variables (25-26) assuming the ranges $x_{l}(t) \in[0,10], x_{2}(t) \in[0,1], x_{3}(t) \in[0,10]$ and $x_{4}(t) \in[0,0.007]$

$$
\begin{aligned}
& M_{1}\left(z_{1}(t)\right)=\frac{z_{1}(t)-a_{2}}{a_{1}-a_{2}}, M_{2}\left(z_{1}(t)\right)=\frac{-z_{1}(t)+a_{1}}{a_{1}-a_{2}}, \\
& N_{1}\left(z_{2}(t)\right)=\frac{z_{2}(t)-b_{2}}{b_{1}-b_{2}}, N_{2}\left(z_{2}(t)\right)=\frac{-z_{2}(t)+b_{1}}{b_{1}-b_{2}},
\end{aligned}
$$

where

$$
a_{1}=\max _{x_{4}(t)} z_{1}(t)=0.9859, \quad a_{2}=\underset{x_{4}(t)}{\min } z_{1}(t)=-0.01,
$$

$$
\begin{array}{cc}
b_{1}=\max _{x_{1}(t), x_{2}(t)} z_{2}(t)=50, & b_{2}=\min _{x_{1}(t), x_{2}(t)} z_{2}(t)=-0.01, \\
c_{1}=\max _{x_{2}(t), x_{3}(t)} z_{3}(t)=9.8039, & c_{2}=\min _{x_{2}(t), x_{3}(t)} z_{3}(t)=-0.01 .
\end{array}
$$

The fuzzy rules for the RF partial model are then:

$$
\begin{gathered}
\text { IF } z_{l}(t) \text { is " } M_{l}\left(z_{l}(t)\right) \text { " and } z_{2}(t) \text { is " } N_{l}\left(z_{2}(t)\right) \text { " } \\
\text { THEN } \dot{x}_{11}^{R F}(t)=A_{11}^{R F} x(t)+B u(t)+d \\
\text { IF } z_{l}(t) \text { is " } M_{l}\left(z_{l}(t)\right) \text { " and } z_{2}(t) \text { is " } N_{2}\left(z_{2}(t)\right) \text { " } \\
\text { THEN } \dot{x}_{12}^{R F}(t)=A_{12}^{R F} x(t)+B u(t)+d \\
\text { IF } z_{l}(t) \text { is " } M_{2}\left(z_{l}(t)\right) \text { " and } z_{2}(t) \text { is " } N_{l}\left(z_{2}(t)\right) \text { " } \\
\text { THEN } \dot{x}_{21}^{R F}(t)=A_{21}^{R F} x(t)+B u(t)+d \\
\text { IF } z_{l}(t) \text { is " } M_{2}\left(z_{l}(t)\right) \text { " and } z_{2}(t) \text { is " } N_{2}\left(z_{2}(t)\right) \text { " } \\
\text { THEN } \dot{x}_{22}^{R F}(t)=A_{22}^{R F} x(t)+B u(t)+d,
\end{gathered}
$$

where the linear subsystems $A_{11}^{R F}, A_{12}^{R F}, A_{21}^{R F}, A_{22}^{R F}$ are derived from

$$
A_{i j}^{R F}=\left[\begin{array}{cccc}
a_{i} q_{o}^{\max }\left(Y_{O 2}-Y_{r} \frac{Y_{O 2}}{Y_{O}}\right)-D & Y_{r} q_{s}^{\max } b_{j} & 0 & 0 \\
a_{i} q_{o}^{\max }\left(-k_{1} Y_{O 2}+k_{2} Y_{r} \frac{Y_{O 2}}{Y_{O}}\right) & -k_{2} Y_{r} q_{s}^{\max } b_{j}-D & 0 & 0 \\
-k_{3} a_{i} Y_{r} q_{o}^{\max } \frac{Y_{O 2}}{Y_{O}} & k_{3} Y_{r} q_{s}^{\max } b_{j} & -D & 0 \\
-k_{5} a_{i} Y_{O 2} q_{o}^{\max } & 0 & 0 & -D
\end{array}\right]
$$

The final aggregated model turns to be:

$$
\begin{aligned}
& \dot{x}^{R F}(t)=\sum_{i=1}^{2} \sum_{j=1}^{2} M_{i}\left(z_{1}(t)\right) N_{j}\left(z_{2}(t)\right)\left\{A_{i j}^{R F} x(t)+B u(t)+d\right\} \\
& y^{R F}(t)=\sum_{i=1}^{2} \sum_{j=1}^{2} M_{i}\left(z_{1}(t)\right) N_{j}\left(z_{2}(t)\right) C x(t)
\end{aligned}
$$

This fuzzy model exactly represents the RF partial nonlinear model in the region $x_{1}(t) \in[0,10], x_{2}(t) \in$ $[0,1], x_{3}(t) \in[0,10]$ and $x_{4}(t) \in[0,0.007]$. The fuzzy exact model for the Rqe1 and Rqe2 models were constructed following the same procedure.

\section{THE EXACT FUZZY OBSERVER}

After an exact fuzzy model for the nonlinear baker's yeast partial model has been obtained, a fuzzy observer can now be designed. The following assumptions are made:

H1. The yield coefficients $k_{1}, k_{2}, k_{3}, k_{4}, k_{5}$ and $k_{6}$ are constant and known.

H2. The ethanol, the dissolved oxygen concentration and the OTR are known.

When the output of the RF partial model is the ethanol and the subsystems given by (31) are used observability full rank cannot be achieved and only is possible to estimate biomass, substrate and ethanol. With this approach dissolved oxygen can not be estimated. However, the observer can still be designed, due to the fact that dissolved oxygen and OTR are usually available on line. The same 
situation applies with the Req1 and Rqe2 partial models. Since the dissolved oxygen is measurable, the premise variable $z_{l}(t)$ is taken as in (25). However for $z_{2}(t)$ and $z_{3}(t)$, we have to consider the estimates, namely

$$
\begin{gathered}
z_{2}(t)=\frac{\hat{x}_{1}}{K s+\hat{x}_{2}} \\
\hat{z}_{3}(t)=\frac{x_{3}}{\left(K e+\hat{x}_{2}\right)\left(K i+x_{3}\right)}
\end{gathered}
$$

To construct the fuzzy observer for the RF partial model the procedure described by Tanaka and Wang (2001) is followed. The membership function for the premise variable $z_{l}(t)$ is once again given by (29); however, for $z_{2}(t)$ the membership function is described by

$$
N_{1}\left(z_{2}(t)\right)=\frac{z_{2}(t)-b_{2}}{b_{1}-b_{2}}, N_{2}\left(\hat{z}_{2}(t)\right)=\frac{-z_{2}(t)+b_{1}}{b_{1}-b_{2}},
$$

To construct the RF observer the linear subsystems $A_{11}^{R F}, A_{12}^{R F}, A_{21}^{R F}, A_{22}^{R F}$ are derived from

$$
A_{i j}^{R F}=\left[\begin{array}{ccc}
a_{i} q_{o}^{\max }\left(Y_{O 2}-Y_{r} \frac{Y_{O 2}}{Y_{O}}\right)-D & b_{j} Y_{r} q_{s}^{\max } & 0 \\
a_{i} q_{o}^{\max }\left(-k_{1} Y_{O 2}+k_{2} Y_{r} \frac{Y_{O 2}}{Y_{O}}\right) & -k_{2} b_{j} Y_{r} q_{s}^{\max }-D & 0 \\
-k_{3} a_{i} Y_{r} q_{o}^{\max } \frac{Y_{O 2}}{Y_{O}} & k_{3} b_{j} Y_{r} q_{s}^{\max } & -D
\end{array}\right]
$$

The fuzzy rules for the RF partial model observer are stated as

$$
\begin{aligned}
& \text { IF } z_{l}(t) \text { is " } M_{l}\left(z_{l}(t)\right) \text { " and } z_{2}(t) \text { is " } N_{1}\left(\hat{z}_{2}(t)\right) \text { " } \\
& \text { THEN } \\
& \hat{\dot{x}}_{11}^{R F}(t)=A_{11}^{R F} \hat{x}(t)+B u(t)+K_{1 \_R F}(y(t)-\hat{y}(t)) \\
& \text { IF } z_{l}(t) \text { is " } M_{1}\left(z_{l}(t)\right) \text { " and } z_{2}(t) \text { is " } N_{2}\left(\hat{z}_{2}(t)\right) \text { " } \\
& \text { THEN } \\
& \hat{\dot{x}}_{12}^{R F}(t)=A_{12}^{R F} \hat{x}(t)+B u(t)+K_{2_{-} R F}(y(t)-\hat{y}(t)) \\
& \text { IF } z_{l}(t) \text { is " } M_{2}\left(z_{1}(t)\right) \text { " and } z_{2}(t) \text { is " } N_{1}\left(\hat{z}_{2}(t)\right) \text { " } \\
& \text { THEN } \\
& \hat{\dot{x}}_{21}^{R F}(t)=A_{21}^{R F} \hat{x}(t)+B u(t)+K_{3 \_R F}(y(t)-\hat{y}(t)) \\
& \text { IF } z_{l}(t) \text { is " } M_{2}\left(z_{1}(t)\right) \text { " and } z_{2}(t) \text { is " } N_{2}\left(\hat{z}_{2}(t)\right) \text { " } \\
& \text { THEN } \\
& \hat{\dot{x}}_{22}^{R F}(t)=A_{22}^{R F} \hat{x}(t)+B u(t)+K_{4 \_R F}(y(t)-\hat{y}(t)),
\end{aligned}
$$

The aggregated fuzzy observer for the RF model turns to be

$$
\begin{aligned}
\hat{\dot{x}}^{R F}(t)=\sum_{i=1}^{2} & \sum_{j=1}^{2} M_{i}\left(z_{1}(t)\right) N_{j}\left(\hat{z}_{2}(t)\right) \\
& \times\left\{A_{i j}^{R F} \hat{x}(t)+B u(t)+K_{i_{-} R F}(y(t)-\hat{y}(t))\right\} . \\
\hat{y}^{R F}(t)= & \sum_{i=1}^{2} \sum_{j=1}^{2} M_{i}\left(z_{1}(t)\right) N_{j}\left(\hat{z}_{2}(t)\right) C \hat{x}(t)
\end{aligned}
$$

The fuzzy observers for the Rqe1 and Rqe2 partial models were derived using the same procedure. The observer gains were calculated by means of the Linear Matrix Inequalities (LMI's) given by (36) and solved using the MATLAB ${ }^{\mathrm{TM}}$ Linear Matrix Inequalities toolbox. The observer gains for the RF, Rqe1 and Rqe2 partial models are shown in table 2.

$$
\begin{aligned}
& X>0 \\
& {\left[\begin{array}{cc}
X & A_{i}^{T} X-C_{i}^{T} N_{i}^{T} \\
X A_{i}^{T}-N_{i}^{T} C_{i} & X
\end{array}\right]>0}
\end{aligned}
$$

where $X=P^{-1}, N=P K, \quad P$ is a common definite positive matrix and $K$ is the observer gain.

Table 2 Fuzzy observer gains for the RF, Rqe1, and Rqe2 partial models.

\begin{tabular}{lccc} 
& $x_{I}$ & $x_{2}$ & $x_{3}$ \\
\hline $\mathrm{K}_{1 \_ \text {RF }}$ & -3009 & 6372 & -57.87 \\
$\mathrm{~K}_{2}$ RF & -5.335 & 8.685 & 0.679 \\
$\mathrm{~K}_{3 \_ \text {RF }}$ & -3004 & 6361 & -58.04 \\
$\mathrm{~K}_{4 \_ \text {RF }}$ & 0.167 & -1.98 & 0.511 \\
$\mathrm{~K}_{1}$ Rqe1 & -1022 & 1996 & 79.14 \\
$\mathrm{~K}_{2 \_ \text {Rqe1 }}$ & -1006 & 1966 & 77.83 \\
$\mathrm{~K}_{3 \_ \text {Rqe1 }}$ & -19.32 & 36.48 & 1.808 \\
$\mathrm{~K}_{4}$ Rqe1 & -3.716 & 6.509 & 0.4972 \\
$\mathrm{~K}_{1}$ Rqe2 & -2093 & 4460 & -56.08 \\
$\mathrm{~K}_{2 \_ \text {Rqe2 }}$ & -3.532 & 5.744 & 0.5116 \\
$\mathrm{~K}_{3 \_ \text {Rqe2 }}$ & -2089 & 4452 & -56.14 \\
$\mathrm{~K}_{4}$ Rqe2 & -0.235 & -1.72 & 0.4531 \\
\hline
\end{tabular}

Also, common positive definite matrices that guarantees global asymptotic stability (Tanaka and Wang, 2001), were found for each partial model, namely

$$
\begin{gathered}
P_{R F}=\left[\begin{array}{ccc}
8.837 \times 10^{-5} & -1.7137 \times 10^{-4} & -2.6913 \times 10^{-6} \\
-1.7137 \times 10^{-4} & 0.36302 \times 10^{-4} & -3.3403 \times 10^{-6} \\
-2.6913 \times 10^{-6} & -3.3403 \times 10^{-6} & 4.962 \times 10^{-6}
\end{array}\right] \\
P_{\text {Rqe1 }}=\left[\begin{array}{ccc}
5.3024 \times 10^{-5} & -1.0182 \times 10^{-4} & -4.4542 \times 10^{-6} \\
-1.0182 \times 10^{-4} & 1.99 \times 10^{-4} & 7.8516 \times 10^{-6} \\
-4.4542 \times 10^{-6} & 7.8516 \times 10^{-6} & 1.0353 \times 10^{-6}
\end{array}\right] \\
P_{\text {Rqe2 }}=\left[\begin{array}{ccc}
2.5022 \times 10^{-3} & -4.9634 \times 10^{-3} & -3.889 \times 10^{-5} \\
-4.9634 \times 10^{-3} & 1.0578 \times 10^{-2} & -1.344 \times 10^{-4} \\
-3.889 \times 10^{-5} & -1.344 \times 10^{-4} & 1.4845 \times 10^{-4}
\end{array}\right]
\end{gathered}
$$

\section{SIMULATION RESULTS}

The application of the proposed observer scheme was simulated using MATLAB ${ }^{\mathrm{TM}}$. The fuzzy observers were tested using the continuous $\mathrm{RF}$ and the $\mathrm{R}$ baker's yeast partial models given above. The inlet substrate concentration was varied between $3 \mathrm{~g} / 1$ and $10 \mathrm{~g} / \mathrm{l}$ in order to force the switching between both models. The initial conditions were chosen as 
$x_{1}(0)=2.0 \mathrm{~g} / 1, \quad x_{2}(0)=0.02 \mathrm{~g} / 1, \quad x_{3}(0)=0.15 \mathrm{~g} / 1$ and $x_{4}(0)=0.0066 \mathrm{mg} / \mathrm{l}$. A white noise signal was added to the ethanol and the dissolved oxygen on line measurements, see figure 2 . The behavior of the fuzzy observer for biomass estimation is shown in figure 3. It can be noticed that the dynamics of the baker's yeast switch through the RF, Rqe1 and Rqe2 partial models. The observer converges to the real biomass values. The estimated substrate is shown in figure 4 , as it can be seen in this case the substrate observer is more sensitive to the white noise signal; however the observer performance is acceptable for the range of chosen values. The rate of convergence could be improved by convenient closed loop eigenvalues relocation.
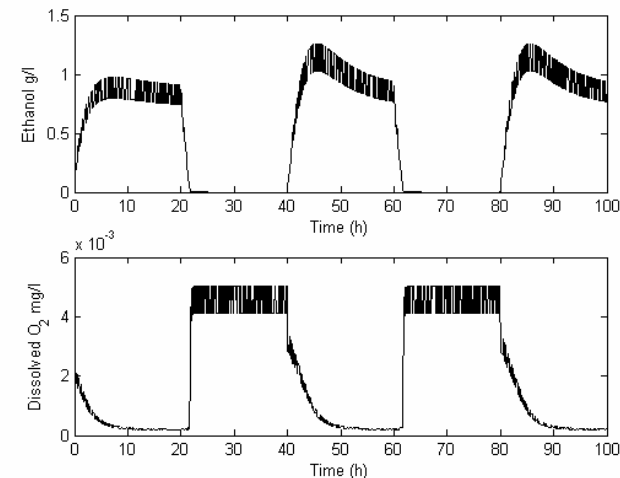

Fig.2. Ethanol and dissolved oxygen with a white noise signal of \pm 0.1 .

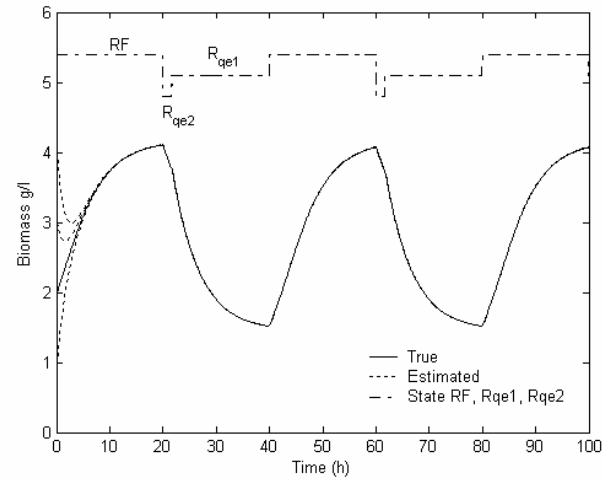

Fig. 3. Biomass estimation for three initial conditions $\hat{x}_{1}(0)=1.0,3.0$ and $4.0 \mathrm{~g} / 1$.

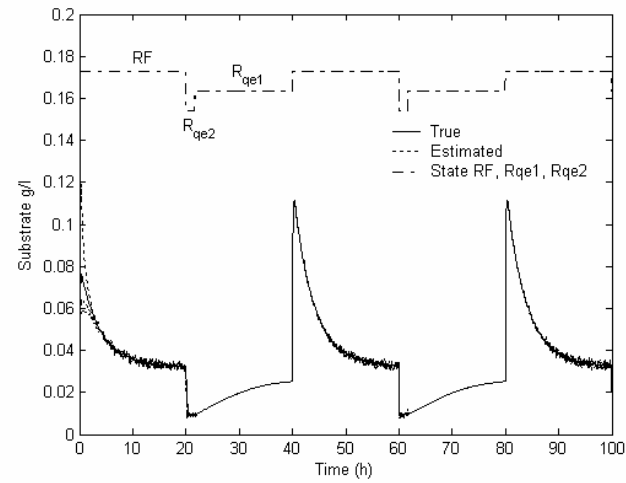

Fig.4. Substrate estimation for three initial conditions $\hat{x}_{2}(0)=0.01,0.03$ and $0.06 \mathrm{~g} / \mathrm{l}$.

\section{CONCLUSIONS}

Based on the idea of splitting the baker's yeast model, a novel TS fuzzy model was proposed using the sector nonlinearities method, giving an exact representation of the original nonlinear plant. Moreover, an observer for each partial model was constructed. It is worth noting that the observer was capable of switching along the partial models, without performance degradation. Therefore, the approach presented here may be considered a valid method to design an observer. Future work will include the experimental validation of the fuzzy observer, as well as the implementation of the fuzzy exact observer for a baker's yeast fed batch culture.

\section{ACKNOWLEDMENTS}

This paper has been supported by the Mexican Consejo Nacional de Ciencia y Tecnología (CONACyT), under grants 46538, 41148 and the $\mathrm{Ph}$. D. scholarship 70662 .

\section{REFERENCES}

Azevedo, S.F., P. Ascencio and D. Sbarbaro (2004). An adaptive fuzzy hybrid state observer for bioprocesses. IEEE Transactions on Fuzzy Systems, 12, 641-651.

Bastin G. and D. Dochain (1990). On-line Estimation and Adaptive Control of Bioreactors. Elsevier science Publishers, Amsterdam.

Dochain, D. (2003). State and parameter estimation in chemical and biochemical processes: a tutorial. Journal of Process Control, 13, 801818.

Ferreira E.C. (1995). Identificação e controlo adaptivo de processos biotecnológicos. Ph. D. Thesis, Universidade do Porto, Portugal.

Horiouchi, J.I. and M. Kishimoto (1998). Fuzzyaided estimation of biological parameters based on material balances. Journal of Fermentation and Bioengineering, 86, 111-117.

Horiouchi, J.I. and M. Kishimoto (2002). Application of fuzzy control to industrial bioprocesses in Japan. Fuzzy Sets and Systems, 128, 117-124.

Pormeleau Y. (1990). Modelisation et controle d'un procéde fed-batch de culture des levures á pain (Saccharommyces cerevisiae). Ph. D. Thesis, Ecole Polytechnique de Montréal, Canada.

Takagi, T. and M. Sugeno (1985). Fuzzy identification of systems and its applications to modelling and control. IEEE Transc. Sys., Man. Cyber. 15, 116-132.

Tanaka K. and H.O. Wang (2001). Fuzzy Control Systems Design and Analysis, A Linear Matrix Inequality Approach. John Wiley \& sons, United States of America. 